\title{
Construção de fonte e cuba de eletroforese horizontal e sua aplicação em aulas práticas de bioquímica
}

\section{Construction of tub and horizontal electrophoresis power and practical application in biochemistry classes}

Renato Ventresqui Oliveira ${ }^{1}$, Joyce Pereira Takatsuka ${ }^{2}$, Vera Lucia Bonfin ${ }^{1}$, Alexandre de Paula Rogério ${ }^{3}$, Afonso Pelli ${ }^{3}$, Pedro Gomes Peixoto ${ }^{1}$.

1- Curso de Graduação em Ciências Biológicas da Universidade Federal do Triangulo Mineiro (UFTM); 2 Curso de Graduação em Ciências Biológicas do Instituto Federal Triangulo Mineiro (IFTM). 3 - Curso de Graduação em Biomedicina da Universidade Federal do Triangulo Mineiro (UFTM).

Endereço para correspondência: Laboratório de Imunofarmacologia Experimental - LIFE. Instituto de Ciências da Saúde. Universidade Federal do Triângulo Mineiro. Rua Vigário Carlos, 162. Uberaba, MG, Brasil 38025-350, Telefone: (34) 3318-5814 - e-mail: alexprogerio@yahoo.com.br

\section{RESUMO}

O presente trabalho apresenta um modelo de eletroforese que pode ser utilizado para 0 ensino de bioquímica proporcionando a compreensão desta técnica pelos estudantes. $O$ objetivo desta eletroforese é oferecer o acesso a esta técnica com custos mais baixos do que o equipamento convencional. A fonte e a cuba foram construídas com componentes eletrônicos simples e de fácil acesso, com a mesma eficiência teórica da fonte e da cuba convencional.

Palavras-chave: Educação, Metodologia de Ensino, Separação de Misturas.

\section{ABSTRACT}

This paper presents a model of electrophoresis for biochemistry classes, were the comprehension of this technique is better absorbed by the students. The aim of this type of electrophoresis is to offer access to this technique with low costs than conventional equipment. The power and the tub were built with electronic parts simple and easy to access, with the same theoretical efficiency of conventional power and tub.

Keywords: Education, Teaching Methodology, Compounds Separation. 


\section{INTRODUÇÃO}

O desenvolvimento de novas metodologias assim como novas estratégias de ensino são necessárias para o melhor aprendizado, compreensão e execução das aulas práticas e teóricas por alunos do ensino médio como, por exemplo, nas aulas de Biologia e Química [1]. A facilidade da aquisição do conhecimento devido à evolução da tecnologia deve estar associada à facilidade de interpretação e compreensão dos temas trabalhados em sala de aula, e para tanto, novas abordagem e estratégias de ensino são necessárias para que as aulas se tornem mais atrativas para os alunos [2]. O uso do conhecimento prático e contextualizado é necessário para o aprendizado [3], não só na educação básica, porém nesta talvez a necessidade seja mais pertinente.

A aula prática como metodologia de ensino favorece a assimilação do conhecimento, facilitando o desenvolvimento de habilidades e aptidões que podem influenciar o aluno suas futuras escolhas profissionais. Especificamente nas disciplinas das áreas de Biologia e Química, o aprendizado prático favorece a familiarização dos alunos com equipamentos e reagentes que poderão estar presentes em seus ambientes de trabalho futuro. Nestas disciplinas, o ensino torna-se complexo, pois alguns temas a serem tratados são abstratos e de difícil entendimento. A finalidade do uso da eletroforese é distinta, porém parte do mesmo princípio de que a técnica propicia a separação de macromoléculas (proteínas, ácidos nucléicos, dentre outras) de diferentes pesos moleculares e isso pode ser visualizado ao se utilizar a técnica.

Além disso, colabora com a sedimentação do conhecimento com trabalho em grupo [4,5].

Na bioquímica. Existem vários tipos de eletroforese, como a eletroforese em gel de poliacrilamida, a eletroforese desnaturante, eletroforese em papel filtro, e a eletroforese capilar. Durante a eletroforese, a amostra a ser analisada é aplicada em "poços" feitos em um gel de corrida que dependendo da amostra tem uma constituição diferente.. O gel de corrida é aplicado em uma cuba de eletroforese preenchida com um tampão para que se tenham condições químicas ideais para a amostra migrar livremente pelo gel e ter seus componentes separados [6].

Sendo assim, este trabalho tem como objetivo construir um modelo didático de uma fonte e cuba de eletroforese de amperagem fixa, para que se possa ensinar o princípio desta técnica de um modo simples e prático ao entendimento dos alunos, proporcionando um conhecimento tanto em ciências, com ênfase em bioquímica e biologia molecular, quanto em eletrônica, pois será enfatizado também o funcionamento da fonte de eletroforese.

\section{DESENVOLVIMENTO}

A fonte de eletroforese que foi construída é um circuito eletrônico em uma placa de circuito impresso. A mesma foi desenhada para obter a ordem correta dos componentes e a passagem da corrente elétrica com a seguinte ordem: (1) quatro diodos IN 400 para

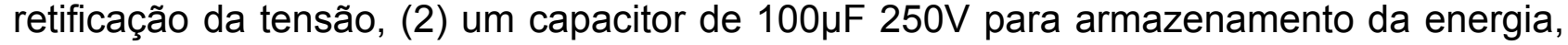
(3) uma resistência de $5 \mathrm{w}$ para limitar a quantidade de energia que sai desse primeiro

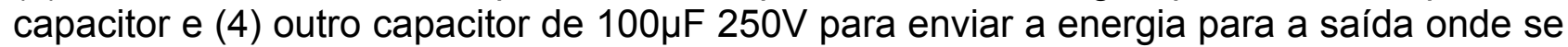
ligarão a energia da cuba de eletroforese.

Depois de realizada a montagem do circuito, ele foi colocado em uma caixa de madeira de $18,5 \mathrm{~cm}$ de comprimento, $11,5 \mathrm{~cm}$ de largura e $3 \mathrm{~cm}$ de altura (Figura 1). Colocou-se um fio com tensão de $130 \mathrm{~V}$ ligada a um fusível de 250 miliamperes limitando a entrada de energia somente a esse valor, prevenindo a queima do aparelho. 


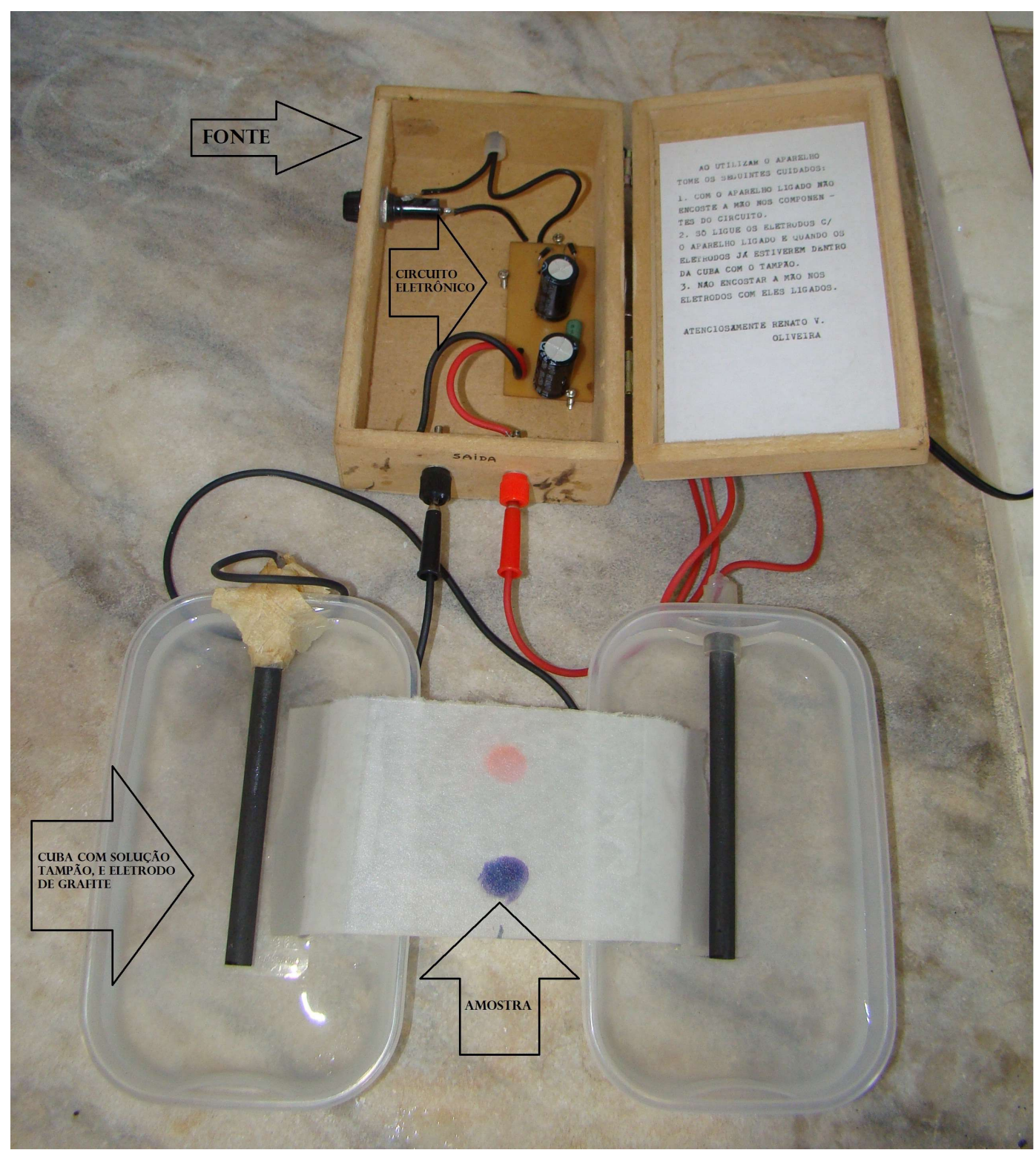

Figura 1. Foto ilustrativa da fonte e da cuba de eletroforese (arquivo pessoal).

$\mathrm{Na}$ saída de energia da caixa foram colocados adaptadores para o acoplamento de pinos banana nas cores: vermelho e preto, sendo o vermelho para a voltagem positiva e o preto, para a negativa (Figura 1).

Duas vasilhas de Plástico foram utilizadas como cubas para que se realize o experimento. Para a montagem das cubas, eletrodos de grafite foram acoplados as duas vasilhas sendo uma destinada para a carga positiva e outra para a carga negativa. (Figura 1).

Para interligar a fonte com as cubas foram utilizados fios de cor vermelha e preta para se adequar aos padrões propostos anteriormente.

Para a utilização do equipamento é necessário que se tenha uma solução de tampão fosfato $0,1 \mathrm{M}$ com $\mathrm{pH} 7,0$. Esta solução tampão é necessária para que haja a condução de corrente elétrica pelo sistema. O uso de um autotransformador Plug in 220V$110 \mathrm{~V}$ modelo 60 foi necessário para a redução da corrente elétrica de127V para $63,5 \mathrm{~V}$.

Como teste, realizou-se um experimento, nas condições descritas acima, utilizando uma fita de papel filtro como suporte e uma mistura de corantes com cargas opostas aplicadas no centro da fita. 


\section{REFLEXÕES}

O modelo de eletroforese proposto como metodologia de ensino, apresentou ótimos resultados nos testes realizados. Durante o experimento, foi possível ver após algum tempo, que o corante com carga positiva, migrou para o eletrodo negativo, e o corante com carga negativa, para o eletrodo positivo (Figura 2). Sendo assim possível a fixação das teorias ensinadas em sala de aula.

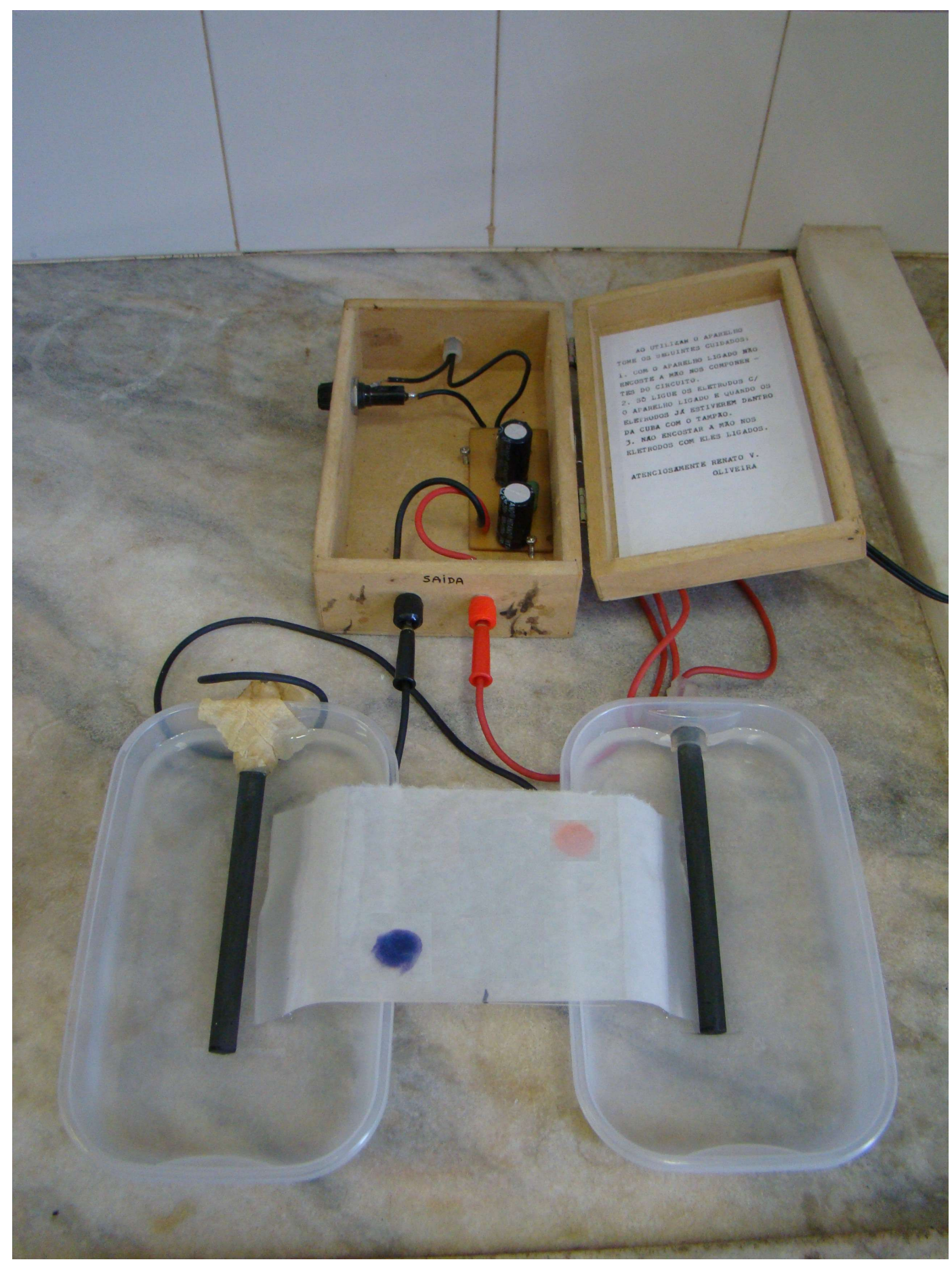

Figura 2. Disposição dos corantes após realização do experimento (arquivo pessoal).

Ao fim do experimento com esse modelo proposto, pôde-se observar que ele apresenta um método mais simples do que as fontes de eletroforese convencionais, para o entendimento da técnica. Conclui-se então, que para a fundamentação do principio da eletroforese pode-se adotar alternativas mais concisas para o ensino da técnica, vindo a propiciar o melhor entendimento aos estudantes. 


\section{REFERÊNCIAS}

[1] BRASIL (2002). Ministério da Educação. Secretaria de Educação Básica. PCN+Ensino Médio: Orientações Educacionais Complementares aos Parâmetros Curriculares para o Ensino Médio: Ciências da natureza, matemática e suas tecnologias. Brasília.

[2] Lei de Diretrizes e Bases da Educação Nacional (LDB) 9.394 de 20/12/1996.

[3] Mckee, E.; Williamson, V. M.; and Ruebush, L. E. (2007) Effects of a demonstration laboratory on student learning, Journal of Science Education and Technology 16.

[4] Niedderer, H.; Aufschnaiter, S.; Tiberghien, A.; Buty, C.; Haller, K.; Hucke, L.; Sander, F.; and Fischer, H. (2002). Talking Physics in laboratory contexts - A category bases analysis of videotapes, In Teaching and Learning in the Science Laboratory (Psillos, D., and Niedderer, H., Eds.), pp 31-50, Kluwer Academic Publishers, London.

[5] PEREZ-SWEENEY, B. M. et al. (2003). Metodologias moleculares utilizadas em genética da conservação. In: CULLEN JR, L., RUDRAN, R. \& VALADARESPADUA, C. (Eds.) Métodos de estudos em biologia da conservação e manejo da vida silvestre. Editora UFPR, Fundação O Boticário de Proteção à Natureza, Curitiba, Paraná, Brasil. 343-380 pp.

[6] YOKAICHIYA, D. K.; GALEMBECK, E. ;; TORRES, B. B. (2001) Expectativas e Fatores de Interesse por Ensino a Distância na área de Bioquímica: relatos de uma pesquisa pré e pós aplicação de uma disciplina de Bioquímica a Distância Revista Brasileira de Ensino de Bioquímica e Biologia Molecular, Artigo 3, Edição 02/2001,Disponível em <http://www.ib.unicamp.br/lte/rbebbm/visualizarMaterial.php? idMaterial=151>. Acesso em: 26 jun. 2012 